\title{
A New Concept for Multi-Beam Phased Array
}

\author{
$1^{\text {st }}$ Boon-Kok Tan \\ Department of Physics (Astrophysics) \\ University of Oxford \\ Oxford OX1 3RH, United Kingdom \\ boonkok.tan@physics.ox.ac.uk
}

\author{
$2^{\text {nd }}$ John D. Garrett \\ Department of Physics (Astrophysics) \\ University of Oxford \\ Oxford OX1 3RH, United Kingdom \\ john.garrent@physics.ox.ac.uk
}

\author{
$3^{\text {rd }}$ Ghassan Yassin \\ Department of Physics (Astrophysics) \\ University of Oxford \\ Oxford OX1 3RH, United Kingdom \\ ghassan.yassin@physics.ox.ac.uk
}

\begin{abstract}
We present a new concept for constructing a compact linear $1 \times n$ multi-beam phased array transceiver system that can be scaled to operate from radio to millimetre wavelength. In this design, all the components required to form the phased array are fabricated on a single printed circuit board, where both the input antenna array and the readout arrays are integrated on the same platform. This is made possible by using a novel planar power splitter/combiners technology, which allows the input signal from each antenna to be split simultaneously through these components arranged in horizontal, and re-combined vertically to form individual beam. This compact single-platform phased array system could be important for many applications that required compact and light-weight design such as 4G/5G wireless telecommunications, inter-satellite (CubeSat) or satellitebase station communication links, space-based remote sensing, vehicle transceiver system and astronomical receivers.
\end{abstract}

Index Terms-Phased arrays, transceivers, microwave circuits, antenna array, beam steering

\section{INTRODUCTION}

An antenna phased array is a transceiver system comprising a series of antennae where the combined beam of the assembly can be steered electronically in different directions without physically moving the antennas. This is done by controlling the phases of the signals received by the antenna array via a phase-shifter network. Multi-beam phased arrays are widely used in many applications, such as $4 \mathrm{G} / 5 \mathrm{G}$ wireless telecommunications [1], satellite applications [2], [3], remote sensing [4], radar imaging system [5], and in astronomy [6]. They can be configured to operate from radio, microwave to millimetre (mm) waves [7], [8] regime. Traditional multibeam phased array systems are bulky and complicated to build, due to the need to split the signals from each antenna element and then re-combine them to form individual beams in a different plane i.e, the antenna array have to be aligned perpendicular to the readout array [9], as shown in Fig. 1(a). This problem however can be alleviated using the novel planar power splitter/combiner technology we reported previously [10], where the signals from two transmission paths can be split and combined simultaneously through this passive circuit element. The planar power splitter/combiner can be fabricated easily using standard planar circuit technology, and hence can be used to replace the complex splitter/combiner networks of a conventional phased array. This reduces significantly the complexity of the array, therefore allowing a much compact and modular array architecture, as shown in Fig. 1(b). Furthermore, this design approach opens up the possibility to construct a 3D multi-beam phased array, where these linear $1 \times n$ arrays can be stacked to form an $n \times n$ 3-D array. This increases the number of the elements from $n$ to $n^{2}$ and allow scanning in two orthogonal planes simultaneously.

This new phased array technology will be highly beneficial for many commercial applications that require compact and light-weight scanning system at microwave frequencies, such as reconfigurable point-to-point communication links, satellite and inter-satellite communication, anti-collision transceiver system for vehicles etc. In the following, we describe the working principle and the experimental measured performances of the planar power splitter/combiner, and how this technology can be applied to develop a new compact multi-beam phased array system.

\section{Planar Cross-Guide Directional Coupler}

The planar signal splitter/combiner is a microstrip crossguide directional coupler that behave exactly like the conventional free space dielectric beam splitter, as illustrated in Fig. 2(a) \& (b). It comprises of two crossing planar circuit transmission lines, with each transmission line fabricated on either side of a dielectric layer. The top layer transmission lines is a microstrip line, while the second transmission line is a coplanar waveguide (CPW) fabricated in the ground plane. The bottom layer CPW is then transformed back to microstrip via a pair of broadside couplers, so that the directional coupler can be integrated easily with the commonly used microstripbased circuits. As can be seen, the directional coupler is fully planar with clearly separated layers, and the entire circuit can be fabricated on a single substrate. A section of the top microstrip is bent and aligned with the central conductor of the bottom CPW (hereinafter the Z-bend section) to increase the power coupling between the crossing transmission lines. An important feature of this alteration is that the inter-arm power coupling is now directional i.e., only a fraction of power from the input port of the $R F 1$ arm (port 1, input) is coupled to the output port of $R F 2$ arm (port 4, coupled), but not the input of $R F 2$ (port 3, isolation). The majority of the RF power would pass through the crossing (port 2, through) and combined with the fractional power coupled from the input of $R F 2$ (port 3 , isolation).

Another key feature of our planar directional coupler is that the coupling coefficient can be controlled easily by altering 

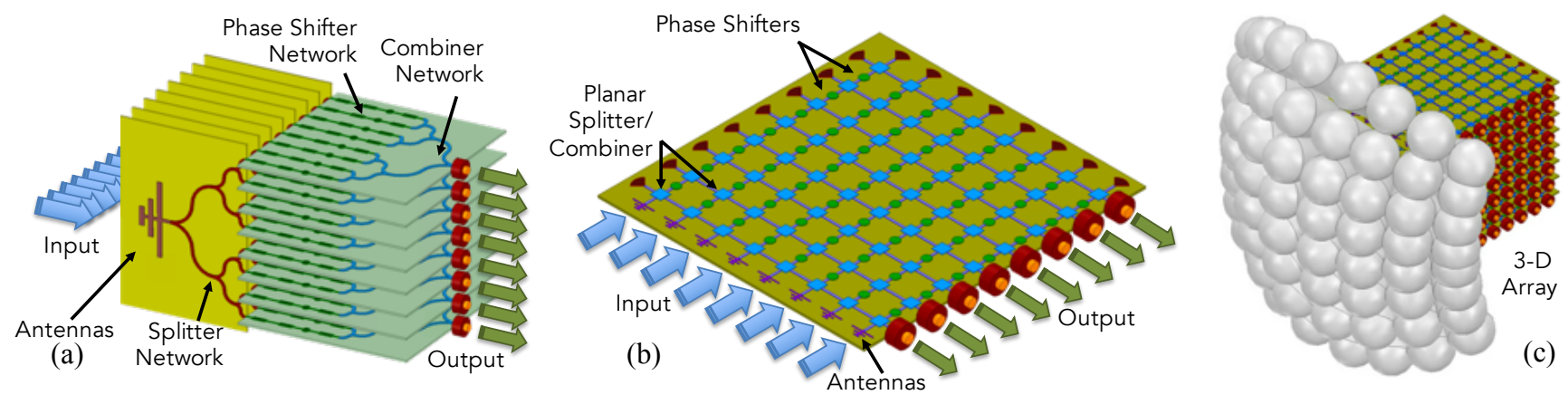

Fig. 1. (a) Traditional linear multi-beam phased array with horizontal input antennas array and vertical readout ports. (b) Proposed single-platform multi-beam phased array, where the input antennas and the readout ports are all in the same plane. (c) A two-dimentional multi-beam phased array.

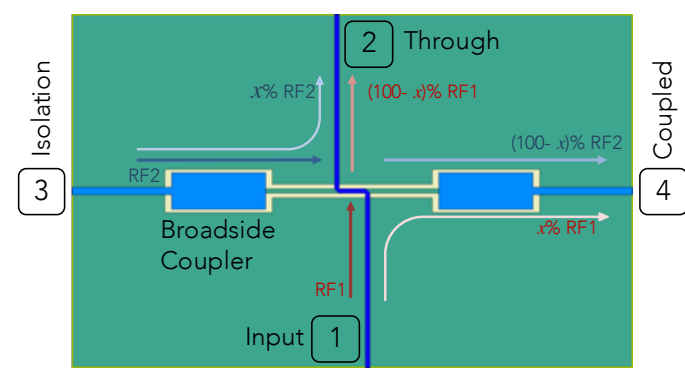

(a)

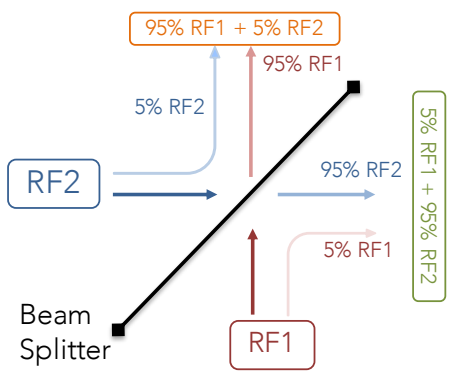

(b)

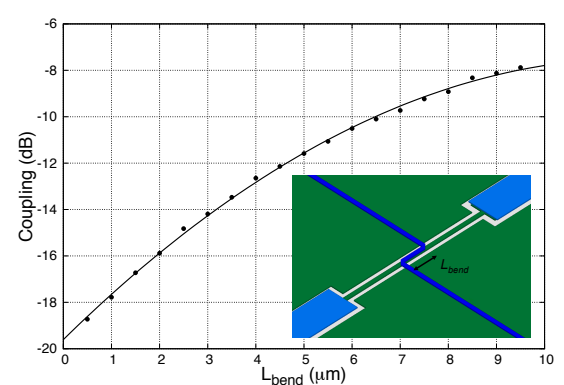

(c)

Fig. 2. (a) A sketch showing the power distribution between the two transmission paths of the directional coupler. (b) Power transmission characteristics of a dielectric beam splitter. (c) The relation between the length of the Z-bend section and the level of power coupling between the two transmission lines.

the length of the Z-bend section without changing other geometries of the circuit. This is illustrated in Fig. 2(c), which shows the power coupling between the two transmission lines that increases approximately exponentially with the length of the Z-bend. This is especially useful for constructing a power distribution network that needs to divide the input power evenly between a series of elements in an array. In particular, the drop of input power after each coupler can be compensated for, by gradually increasing the Z-bend length, ensuring the input power delivered to all element remains constant.

In order to test the performance of the planar cross-guide directional coupler, we have designed and fabricated several prototypes operating around $12.5 \mathrm{GHz}$ band, with six different coupling coefficients. Fig. 3(a) shows a photograph of one of the assembled prototypes. The directional couplers were fabricated on both side of a 10-mil thin Roger RO4350 ${ }^{\mathrm{TM}}$ printed circuit board (PCB), supported by a thicker Roger Duroid 6010 bare substrate. The S-parameters behaviour of all four ports of the device were measured simultaneously using a fourport VNA. The results for all six prototypes are summarised in Fig. 3(c) and (d). The measured return loss for both transmission arms is below $-15 \mathrm{~dB}$ from $12-14 \mathrm{GHz}$, and the isolation is close to $-20 \mathrm{~dB}$ across the entire $11-14 \mathrm{GHz}$ band. These results agree very well with simulation. The closely separated coupling levels for the six prototypes can clearly be identified and remain relatively flat across the band. The dependence of the coupling coefficient on the length of the Z-bend is plotted in Fig. 3(b), which shows that the measured coupling coefficients is indeed agree very well with the simulated curve, where the coupling responses increased approximately exponentially with the Z-bend length.

\section{Applications to Phased-ArRay System}

The top panel of Fig. 4 shows how the conventional phased array system is normally constructed. The input signal from each antenna elements is split into several channels before the phase shifters networks. These split signals are then fed into a complicated crossing wiring network in order to distribute them to individual readout ports that combine these signals from each antenna to form a single beam pattern in a particular direction. As can be seen, this involves complicated splitting network, complex inter-connecting crossing wiring network, and a separated combiner network. Due to the need for the crossing wiring network, these phased array systems must have the input antenna array fabricated in perpendicular to the readout port. In other words, a 3-D system for a linear one-dimensional beam.

Owing to the capability of our planar cross-guide directional coupler that can split and combine RF signals simultaneously, we can therefore replace these complicated splitter, cross wiring and combiner networks with single directional coupler grid, significantly reducing the complexity of the multi-beam phased-array system. This can be achieved using a 2-D grid of the couplers, as described in the bottom panel of Fig. 4. Here, both the input antenna array and the readout arrays are in the 


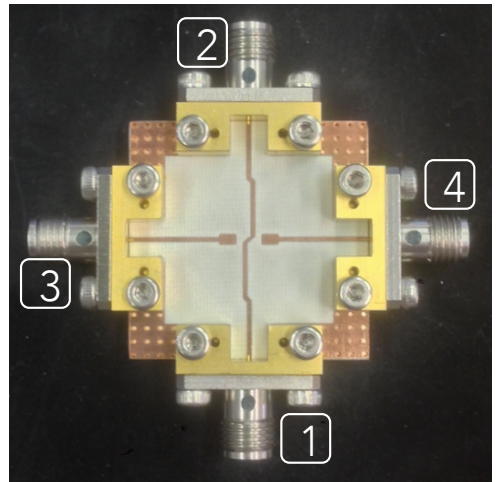

(a)

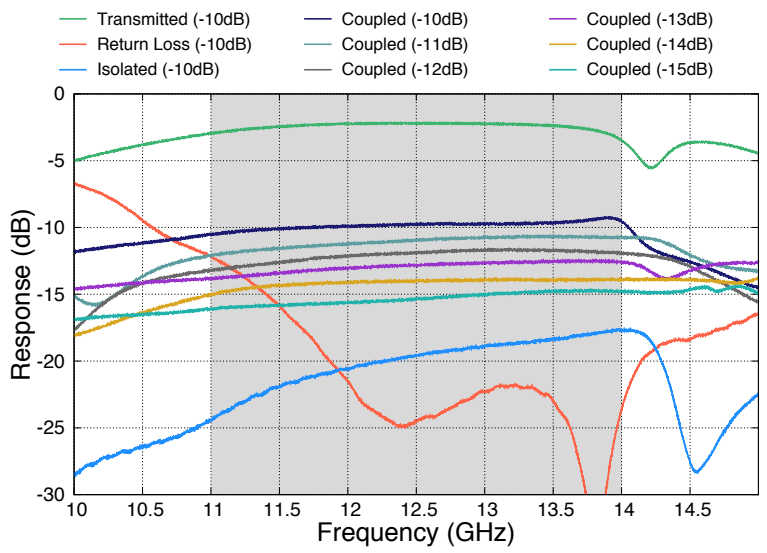

(c)

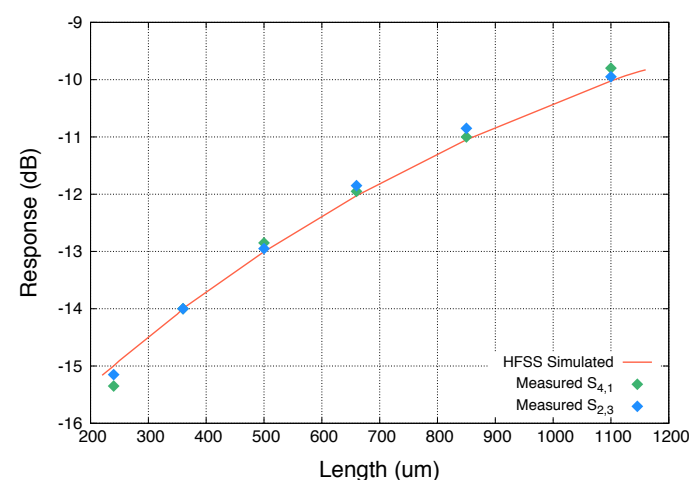

(b)

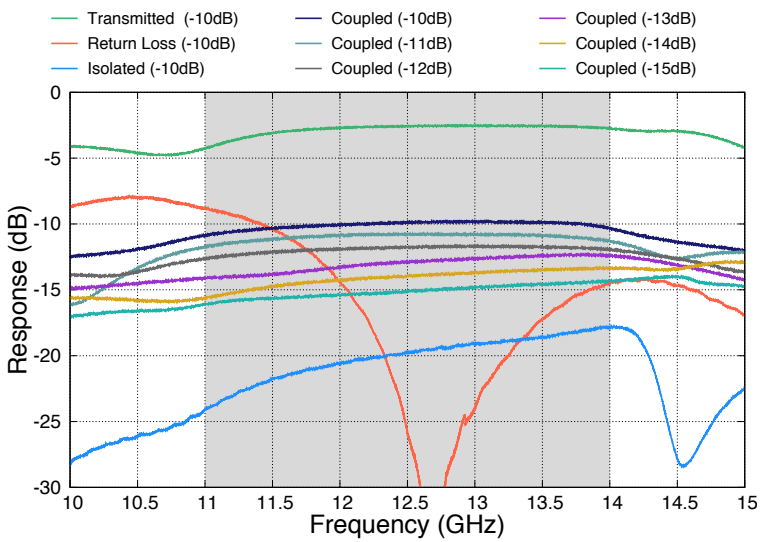

(d)

Fig. 3. (a) Photograph of an assembled directional coupler prototype. (b) The measured relation between Z-bend length and power coupling between $R F 1$ \& $R F 2$. (c-d) The measured transmission, return loss, isolation and coupling coefficients from (c) the input port (1) of $R F 1$, and (d) the input port (3) of $R F 2$.

same plane, where the input signal from each antenna is split through the directional coupler arranged in horizontal, and at the same time, the signal from each antenna are combined to form individual beam vertically via the similar sets of coupler. In the example shown, an 8-element phased array with 8 individual beams would require 480 splitting/combining knots in the existing technology, whereas our planar phased array only requires 64 directional couplers, almost an order of magnitude less. Phase shifter circuits could be installed between columns of the coupler network to steer the beam in the desired direction, similar to the standard approach.

It is important to note that since our cross-guide directional coupler is a planar circuit element, all the input/output array can be formed on the same plane (i.e., on a single PCB), hence offering the opportunity to further form a 2-D beam array by simply stacking these single-platform linear phased array vertically. This would allow the beam to be steered at any direction, instead of just along the antenna array plane, providing more flexibility and faster mapping capability to the system. A 2-D phased array would be extremely difficult to construct using traditional technology, since a single-plane array is already in the form of 3-D architecture as shown previously in Fig. 1(a).
A suitable antenna for the proposed planar phased array would be the dual-dipole antenna that we have developed perviously. This antenna has twice the bandwidth of the conventional Yagi antenna, while retaining a simple geometry. Fig. 5(a) shows the layout of our broadband planar dualdipole antenna (the details of the design can be found in [11]). In Fig. 5(b), we show a prototype dual-dipole antenna designed and fabricated at $15 \mathrm{GHz}$. Here, we cascaded two dipoles of different lengths in series to increase the bandwidth. Two printed directors and a truncated ground plane are used to achieve high front-to-back (F/B) gain ratio. The parallel stripline feeding the antenna's drivers is connected directly to microstrip without an intermediate stage, so that it can be integrated easily with microstrip circuits. In Fig. 5(c)-(d), we show the measured radiation patterns of the antenna fabricated on a Roger RO 4350 PCB. The antenna has $7 \mathrm{GHz}$ bandwidth and a F/B gain ratio of $\sim 18 \mathrm{~dB}$. The measured beam patterns agreed very well with the electromagnetic simulations.

\section{CONCLUSION}

We have presented a new concept for building a compact linear phased-array system on a two-dimensional platform, that can be used to replace the conventional bulky and complicated system setup, which are expensive and difficult 

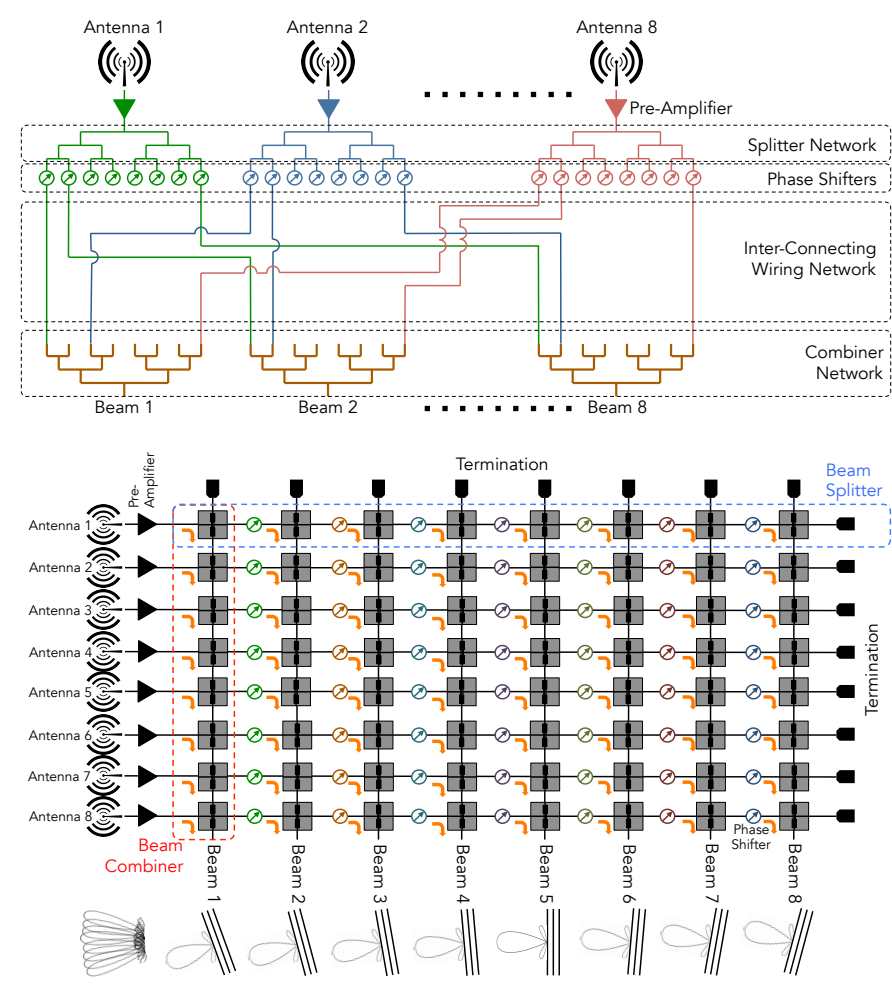

Fig. 4. (Top) Traditional linear multi-beam phase array architecture. (Bottom) Proposed planar linear multi-beam phase array architecture.

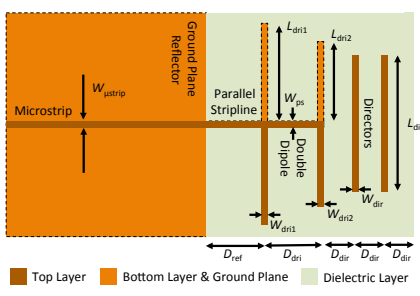

(a)

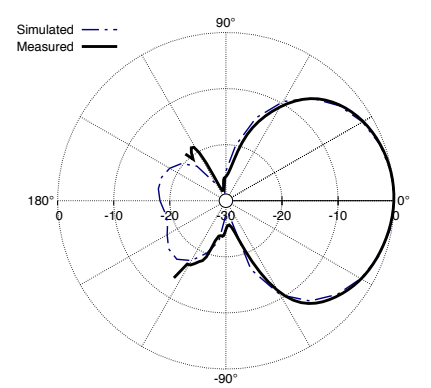

(c) $15 \mathrm{GHz}$ E-plane

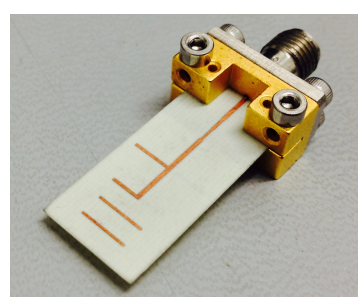

(b)

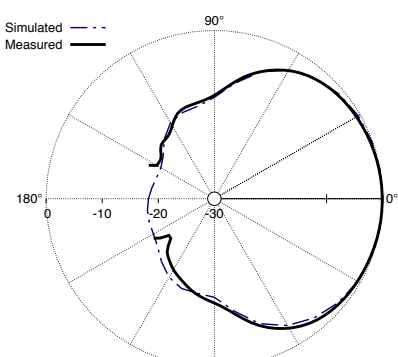

$-90^{\circ}$

(d) $15 \mathrm{GHz}$ H-plane
Fig. 5. (a) Layout of the planar circuit antenna utilising a pair of dipole drivers, and the critical dimensions dictating the performance of the antenna. (b) A Ku-band dual-dipole antenna fabricated on a $254 \mu \mathrm{m}$ thick Roger RO 4350 PCB. (c-d) E- and H-plane far-field radiation patterns of the antenna measured (solid line), along with the simulated beam patterns (dashed line). to construct. This is achieved by substituting the complex splitter, cross-wiring and combiner network in the traditional approach with a simpler planar circuit cross-guide directional coupler grid. This coupler network and the accompanying components required to form the phased array, including the phase shifters and the microstrip antennas, are integrated on a single PCB substrate. We have tested the planar crossguide coupler and the microstrip antenna, and the measured performances match very well with simulation results, with about $24 \%$ bandwidth around $12.5 \mathrm{GHz}$ band. This novel phased array design could potentially be important for many applications that requires miniaturised and light-weight design, in particular the emerging wireless telecommunications and inter-satellite/satellite-base station communication links.

\section{REFERENCES}

[1] W. Hong, Z. H. Jiang, C. Yu, J. Zhou, P. Chen, Z. Yu, H. Zhang, B. Yang, X. Pang, M. Jiang, et al., "Multibeam antenna technologies for 5g wireless communications," IEEE Transactions on Antennas and Propagation, vol. 65, no. 12, pp. 6231-6249, 2017.

[2] A. Jacomb-Hood and E. Lier, "Multibeam active phased arrays for communications satellites," IEEE Microwave Magazine, vol. 1, no. 4, pp. 40-47, 2000.

[3] J. Li, Z. Zhou, X. Chen, J. Zhang, S. Wang, and R. Wu, "Ka band multi-beam phased array antenna for communication satellite application," in 2016 IEEE Advanced Information Management, Communicates, Electronic and Automation Control Conference (IMCEC), pp. 209-213, IEEE, 2016.

[4] R. Zahn and E. Schmidt, "A phased array bread board for future remote sensing applications," in Quantitative Remote Sensing: An Economic Tool for the Nineties, Volume 1, pp. 2269-2272, 1989.

[5] B. Isom, R. Palmer, R. Kelley, J. Meier, D. Bodine, M. Yeary, B.-L. Cheong, Y. Zhang, T.-Y. Yu, and M. I. Biggerstaff, "The atmospheric imaging radar: Simultaneous volumetric observations using a phased array weather radar," Journal of Atmospheric and Oceanic Technology, vol. 30, no. 4, pp. 655-675, 2013.

[6] G. W. Kant, P. D. Patel, S. J. Wijnholds, M. Ruiter, and E. van der Wal, "Embrace: A multi-beam 20,000-element radio astronomical phased array antenna demonstrator," IEEE Transactions on Antennas and Propagation, vol. 59, no. 6, pp. 1990-2003, 2011.

[7] G. Toso, P. Angeletti, and C. Mangenot, "Multibeam antennas based on phased arrays: An overview on recent esa developments," in The 8th European Conference on Antennas and Propagation (EuCAP 2014), pp. 178-181, IEEE, 2014.

[8] L. Schulwitz and A. Mortazawi, "A compact dual-polarized multibeam phased-array architecture for millimeter-wave radar," IEEE Transactions on Microwave Theory and Techniques, vol. 53, no. 11, pp. 3588-3594, 2005.

[9] W.-Y. Chen, Y.-R. Hsieh, C.-C. Tsai, Y.-M. Chen, C.-C. Chang, and S.F. Chang, "A compact two-dimensional phased array using grounded coplanar-waveguides butler matrices," in 2012 42nd European Microwave Conference, pp. 747-750, IEEE, 2012.

[10] B.-K. Tan and G. Yassin, "A planar beam splitter for millimeter and submillimeter heterodyne mixer array," IEEE Transactions on Terahertz Science and Technology, vol. 7, no. 6, pp. 664-668, 2017.

[11] B.-K. Tan, S. Withington, and G. Yassin, "A Compact Microstripfed Planar Dual Dipole Antenna for Broadband Applications," IEEE Antenna and Wireless Propagation Letter, vol. 15, pp. 593-596, july 2015. 